\title{
DRGs - eine Chance für Spitäler
}

\author{
Als Antwort auf die Frage von Berchtold und Schmitz nach der Optimierungsfähig- \\ keit klinischer Prozesse stellt dieser Beitrag eine Studie vor, mit der ein Modell ent- \\ wickelt werden soll, das zeigt, wie SwissDRGs Pflegekontextfaktoren beeinflussen. \\ Ergebnisse eines solchen Monitorings können zur Steuerung und zur Entwicklung \\ klinischer Prozesse beitragen.
}

\begin{abstract}
Michael Kleinknecht ${ }^{a}$, Diana Staudachera, Irena Anna Freic, Elisabeth Spichiger ${ }^{b, d}$, Jacqueline S. Martinc, Rebecca Spiriga, $b$
\end{abstract}

a Projektleiter DRG Begleitforschung Pflege, Zentrum Klinische Pflegewissenschaft, UniversitätsSpital Zürich

b Institut für Pflegewissenschaft, Universität Basel

c Abteilung Klinische Pflegewissenschaft, Universitätsspital Basel

d Bereich Fachentwicklung und Forschung, Direktion Pflege/MTT, Inselspital Universitätsspital Bern

Korrespondenz:

Michael Kleinknecht, MNS Zentrum Klinische Pflegewissenschaft UniversitätsSpital Zürich Rämistrasse 100 (ZUR 44) CH-8091 Zürich Tel. 0442553882 Fax 0442554395

\section{Einleitung}

Grosse Integrationsarbeit steht den Spitälern bevor. Mit der Einführung der SwissDRGs sind medizinische Leistungserbringer gefordert, Behandlungsprozesse stärker als bisher gemeinsam mit Wirtschaftsexperten festzulegen. Klinische Prozesse charakterisieren sich dadurch, dass sie hochgradig komplex und «unberechenbar» sind, betonen Berchtold und Schmitz in ihrem Artikel «Eine Zukunft für Spitäler» [1]. Diese kaum vorhersagbare Vielschichtigkeit stellt in ihren Augen eine Herausforderung dar, die sich mit klassischen Optimierungsbemühungen kaum befriedigend lösen lässt. Dies könnte, so die Autoren, ein Hauptziel der Einführung von SwissDRG gefährden: die Optimierung klinischer Prozesse.

Es stellt sich also die Frage, welche alternativen Optimierungsansätze wir brauchen, um Behandlungsprozesse im Spital effektiver zu gestalten.

In diesem Zusammenhang richtet eine Gruppe von Pflegewissenschaftlerinnen und -wissenschaftlern im Vorfeld der Einführung von SwissDRG ihr Augenmerk auf mögliche Faktoren, die den strukturellen Kontext der Pflegeleistungen abbilden und als solche deren Qualität und Ergebnis mutmasslich mitbestimmen, wie Komplexität der Pflege, pflegerische Kompetenz, professionelle Autonomie und Ressourcen, Leadership, Stellenbesetzung, Arbeitsumgebungsqualität usw. Sie stellen damit einen Ansatzpunkt für Optimierungsstrategien dar, um Leistungen in einem Spital zweckmässig, wirksam und zugleich wirtschaftlich zu erbringen [2-5]. Qualität und Effektivität pflegerischer Leistungen werden wesentlich unterstützt, wenn solche Kontextfaktoren positiv beeinflusst und dadurch notwendige Rahmenbedingungen sichergestellt werden [6-9]. Die internationale Forschung hat sich mit dieser Thematik schon länger auseinandergesetzt und ist dabei zu Ergebnissen gekommen, die sich im Zeitalter von SwissDRG als unterstützend erweisen können: In verschiedenen Studien wurde nachgewiesen, dass diese Kontextfaktoren einen Effekt auf die Pflegequalität, die Verweildauer oder die Patientensterblichkeit haben [10-13].

\section{DRG - une chance}

En réponse à la question de Berchtold et Schmitz concernant la capacité d'optimiser les processus cliniques, le rapport ci-dessous présente une recherche ayant pour but de développer un modèle de monitoring, permettant de suivre de manière continue l'impact des DRG sur les facteurs contextuels relatifs aux prestations de soins infirmiers dans les hôpitaux suisses. Les données d'un tel monitoring pourraient contribuer à la compréhension multiprofessionnelle ainsi qu'à la mise en place de concepts de processus cliniques et de traitements.

\section{Schritte zu einem multiprofessionellen Verständnis klinischer Leistungserbringung}

Wie wirken sich SwissDRGs auf diese Pflegekontextfaktoren eines Schweizer Spitals aus? Wenn die Entwicklung aufgrund von SwissDRGs mit Hilfe eines kontinuierlichen Monitorings an Spitälern nachverfolgt werden kann, ist dies ein bedeutsamer Schritt, denn die meisten Akutspitäler der Schweiz führen bisher noch kein Monitoring von versorgungsrelevanten Kontextfaktoren durch. Somit fehlen bis heute jene Daten, auf welche die Spitaldirektionen in Zukunft angewiesen sind, um Ressourcen effektiv zu verteilen. Genau hier setzt die von 2011 bis 2013 andauernde Studie «Monitoring the Impact of the DRG-Payment System on Nursing Service Context Factors in Swiss Acute Care Hospitals» an. Sie bildet den pflegewissenschaftlichen Beitrag in einem multiprofessionellen, vom Schweizerischen Nationalfonds geförderten Sinergia-DRG-Begleitforschungsprojekt. Ziel der Pflegestudie ist es, ein Modell für ein Monitoringsystem pflegerelevanter Kontextfaktoren in Schweizer Spitälern aufzubauen. Unter der Leitung des Zentrums Klinische Pflegewissenschaft des UniversitätsSpitals Zürich soll 
das Monitoring gemeinsam mit den Universitätsspitälern Basel und Bern und dem Institut für Pflegewissenschaft der Universität Basel auf der Basis von früheren Studien aufgebaut werden. Das Vorliegen dieses die Möglichkeit, den von Berchtold und Schmitz angeführten «clinical divide» zu überwinden zugunsten eines multiprofessionellen, interdisziplinären Prozessdenkens und -handelns, um gemeinsame professio-

\section{Monitoringsysteme können einen wichtigen Beitrag dazu leisten, dass Patienten auch im DRG-Zeitalter weiterhin qualitativ hochstehend behandelt werden können.}

\begin{abstract}
Monitoringsystems kann Beitrag zu einer weiteren und bedeutenden Chance sein: Mit den sich daraus ergebenden Daten kann im Kontext der SwissDRG Einführung die Entwicklung eines multiprofessionellen Verständnisses klinischer Leistungserbringung und Behandlungskonzepte unterstützt werden, die neben medizinischen und pflegerischen Perspektiven auch ökonomischen Aspekte berücksichtigt [14, 15].

Der Beginn des DRG Zeitalters in der Schweiz kann also einen neuen Abschnitt markieren. Wann, wenn nicht jetzt mit der Einführung der DRG, bietet sich
\end{abstract}

\section{Kurzüberblick Studie «Monitoring the Impact of the DRG-Payment System on Nursing Service Context Factors in Swiss Acute Care Hospitals».}

\section{Hintergrund}

Die DRG-Einführung hat in anderen Ländern gezeigt, dass die Spitäler dadurch einen kleineren finanziellen Spielraum erhalten und eine Restrukturierung der Leistungen unumgänglich wird. Seitens der Pflege können Restrukturierungen zu einem Abbau von Personal und Pflegeleistungen führen, welche die Pflegequalität und Patientenergebnisse negativ beeinflussen.

Um auch in Zukunft eine kompetente Pflege anbieten zu können und die Pflegequalität zu sichern, sind im Sinne eines kontinuierlichen Monitoring Erhebungen von Pflegekontextfaktoren und deren Einfluss auf Pflegequalität und Patientenresultate vonnöten. Unter Pflegekontextfaktoren werden Umgebungsvariablen verstanden, die die Pflegequalität und pflegesensitive Patientenergebnisse wesentlich beeinflussen.

Unter der Führung des Zentrums Klinische Pflegewissenschaft des UniversitätsSpitals Zürich werden die drei Universitätsspitäler Basel, Bern und Zürich sowie das Kantonsspital Winterthur und die Solothurner Spitäler AG als Teilprojekt einer vom Nationalfonds unterstützten multiprofessionellen SNF Sinergia Studie (CRSII3_132786 / 1) noch vor der DRG-Einführung ein erstes Monitoring durchführen.

\section{Zielsetzung}

Die Studie hat zum Ziel, dieses Monitoringsystem zur kontinuierlichen Überwachung des Einflusses der DRG-Finanzierung auf die Pflegekontextfaktoren mitsamt den dazugehörenden Instrumenten zu entwickeln.

\section{Methode}

Mit der deskriptiv-explorativen Evaluationsstudie werden in einem Mixed-Methods-Design in den genannten fünf Spitälern im Sommer 2011 mit Online-Fragebogen auf etwa 190 Abteilungen bei Pflegefachleuten quantitative Daten gesammelt. Im Frühjahr 2012 werden diese durch die Erkenntnisse aus mehreren Fokusgruppeninterviews mit Pflegefachleuten durch qualitative Daten ergänzt. Bis zum Winter 2012 werden die quantitativen und qualitativen Ergebnisse ausgewertet und integriert, so dass in einer ersten Version Ende 2012 das Monitoringsystem und die dazugehörenden Beurteilungsinstrumente in einer ersten Version zur Verfügung stehen sollten. Im Verlaufe 2013 wird dieses verfeinert und weiterentwickelt, so dass es danach für die Überführung in die betriebliche Organisation zur Verfügung stehen wird. nelle Zielsetzungen und ethische Werthaltungen zu entwickeln und zusammen im Sinne der Patientinnen und Patienten beste Behandlungsergebnisse zu erreichen [16-18]?

\section{Das «Spital der Zukunft»}

Prozesse der klinischen Leistungserbringung zu optimieren, muss nicht zwangsläufig nur bedeuten, diese zu standardisieren und die Vorgaben unmittelbar auf die individuelle Patientensituation anzuwenden, wie dies Berchtold und Schmitz beschrieben haben. Optimieren kann auch heissen, die Leistungserbringung neben der Fokussierung auf die individuellen Bedürfnisse der Patientinnen und Patienten verstärkt auf die gesundheitspolitischen und gesellschaftlichen Erwartungen abzustimmen $[19,20]$.

Das «Spital der Zukunft» wird aufgrund der vielfältigen Entwicklungen wohl von der Leistungserbringung der medizinischen, pflegerischen und ökonomischen Professionellen geprägt sein. Eine Verständigung kann leichter gelingen, wenn medizinische, pflegerische, berufsethische und ökonomische Prinzipien und Handlungskonzepte als integratives Ganzes verstanden werden [21, 22]. Professionelle Höchstleistungen werden so mit wirtschaftlichen und gesellschaftlichen Anforderungen vereinbar und damit auch erreichbarer werden.

Um die entsprechend notwendigen Diskussionen $\mathrm{zu}$ führen und die Leistungserbringung nicht nur strategisch auszurichten, sondern auch operativ zu planen, zu steuern und zu kontrollieren, sind entsprechende Kennzahlen notwendig. Ergänzend zu dem beschriebenen Monitoringsystem bezüglich des Einflusses der DRGs auf pflegerelevante Kontextfaktoren, entwickelt und führt z. B. das UniversitätsSpital Zürich derzeit ein «DRG-Steuerungscockpit» ein, das als übersichtliches Steuerungsinstrument Daten für Spitaldirektion, Medizinbereiche und Kliniken zur Optimierung der Leistungserbringung unter möglichst «idealen» Bedingungen und Rahmenparametern mit maximaler Wirtschaftlichkeit und höchstmöglicher Qualität liefern soll [23].

Gelingt es, nicht nur die Daten aus solchen verschiedenen Monitoringsystemen zu einem Ganzen zu synthetisieren, sondern mit deren Hilfe auch die damit verbundenen unterschiedlichen professionellen 


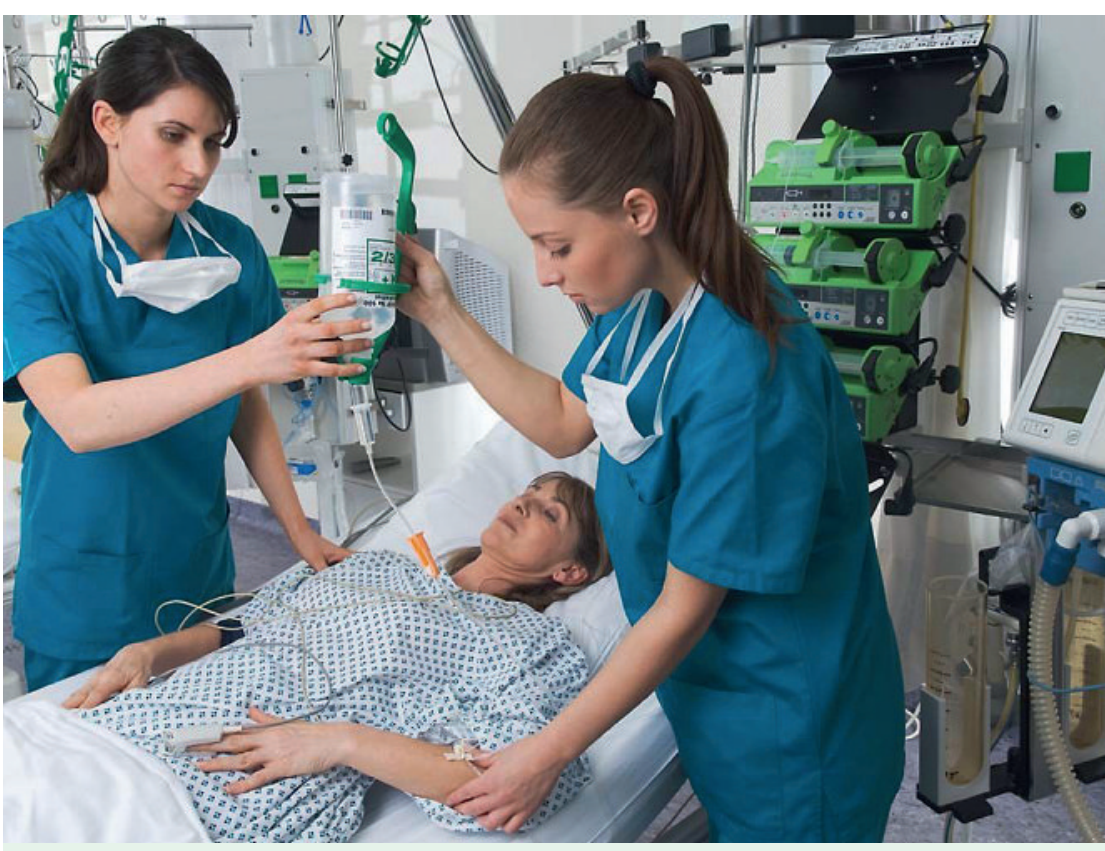

Wie wirkt sich die DRG-Finanzierung auf die Pflege aus? Im Rahmen einer Studie sollen Monitoringsysteme entwickelt werden, um diese Frage beantworten zu können.

Perspektiven und Handlungsmaximen gegenseitig verständlich zu machen, ist wohl ein ganz wesentlicher Schritt in die Richtung getan, auf dieser Basis entsprechende integrierende, multiprofessionelle Strategien und inhaltliche Leistungserbringungskonzepte zu entwickeln.

Dabei können Monitoringsysteme einen wichtigen Beitrag dazu leisten, die professionellen Rahmenbedingungen so zu gestalten, dass Patienten auch im DRG-Zeitalter weiterhin qualitativ hochstehend, individuell und zugleich wirtschaftlich effizient behandelt werden können [24].

\section{Literatur}

1 Berchtold P, Schmitz C. Eine Zukunft für Spitäler. Schweiz Ärztezeitung. 2010;91(48):1914-16.

2 Capuano T, Bokovoy J, Hitchings K, Houser J. Use of a validated model to evaluate the impact of the work environment on outcomes at a magnet hospital. Health Care Manage Rev. 2005;30(3):229-36.

3 Schmalenberg C, Kramer M. Essentials of a productive nurse work environment. Nursing Research. 2008; 57(1): 2-13.

4 Needleman J, Kurtzman ET, Kizer KW. Performance measurement of nursing care. Medical Care Research and Review. 2007; 64(2 Supplementum):10S-43S.

5 Mark BA. Sayler J, Smith CS. A theoretical model for nursing systems outcome research. Nursing Administration Quarterly. 1996; 20(4): 12-27.

6 Aiken LH, Clarke SP, Sloane DM. Effects of hospital care environment on patient mortality and nurse outcomes. JONA. 2008;38(5):223-9.
7 Mark BA, Salyer J, Wan TTH. Professional nursing practice; Impact on organisational and patient outcomes. JONA. 2003;33(4):224-34.

8 Irvine Doran D, Sidani S, Keatings M, Doidge D. An empirical test of the Nursing Role Effectiveness Model. J Adv Nurs. 2002;38(1):29-39.

9 Currie V, Harvey G, West E, McKenna H, Keeney S. Relationship between quality of care, staffing levels, skill mix and nurse autonomy: literature review. J Adv Nurs. 2005;51(1):73-82.

10 Martin JS, Frei IA, Suter-Hofmann F, Fierz K, Schubert M, Spirig R. Evaluation der Pflegeund Führungskompetenz - eine Ausgangslage für die weitere Praxisentwicklung. Pflege. 2010;23(3):191-203.

11 Mitchell PH, Lang NM. Framing the problem of measuring and improving healthcare quality, has the quality health outcomes model been useful? Medical Care. 2004;42(2):4-11.

12 Rafferty AM, et al. Outcomes of variation in hospital nurse staffing in English hospitals: cross-sectional analysis of survey data and discharge records. Int J Nurs Stud. 2007. 44(2):175-82.

13 Aiken LH, Clarke SP, Sloane DM. Hospital staffing, organization, and quality of care: cross-national findings. Int J Qual Health Care. 2002;14(1):5-13.

14 McCormick K. Future data needs for quality of care monitoring, DRG considerations, reimbursement and outcome measurement. Journal of Nursing Scholarship. 1991;23(1):29-32.

15 Heslop L, Gardner B, Athan D, Diers D, Taylor C. Researching hospital patient data to enhance operational management. Health Information Management. 2004;32(1):13-6.

16 Biller-Andorno N, Lenk C, Leititis J. Ethics, EBM, and hospital management. Journal of Medical Ethics.2004;30:136-40.

17 Wehkamp KH. DRGs als medizinethische Herausforderung. Deutsche Medizinische Wochenschrift. 2002;127(8):395-8.

18 Torjuul K, Sorlie V. Nursing is different than medicine: ethical difficulties in the process of care in surgical units. J Adv Nurs. 2006;56(4):404-13.

19 Rycroft-Malone J, Seers K, Titchen A, Harvey G, Kitson A, McCormack B. What counts as evidence in evidence-based practice? J Adv Nurs. 2004;47(1): 81-90.

20 Fineout-Overholt E, Johnston L. Teaching EBP: implementation of evidence: moving from evidence to action. Worldviews on Evidence-Based Nursing. 2006 (Fourth Quarter);194-200.

21 Hamric AB, Blackhall LJ. Nurse-physician perspectives on the care of dying patients in intensive care units: collaboration, moral distress, and ethical climate. Critical Care Medicine. 2007;35(2):422-9.

22 Wehkamp KH. Brücke zwischen Qualität und Ökonomie. Deutsches Ärzteblatt. 2004;101(36):A2374-8.

23 UniversitätsSpital Zürich. DRG@USZ. 2010, UniversitätsSpital Zürich: Zürich.

24 Norrish BR, Rundall TG. Hospital restructuring and the work of registered nurses. Milbank Q. 2001;79(1): 55-79 IV. 